\title{
Desemprego e suicídio na população brasileira em um cenário de crise do capitalismo
}

\author{
Unemployment and suicide among the Brazilian population \\ in the crisis of capitalism
}

Antonio Angelo Menezes Barreto (https://orcid.org/0000-0002-5917-9135) ${ }^{1}$

Luis Eugenio Portela Fernandes de Souza (http://orcid.org/0000-0002-3273-8873) ${ }^{1}$

${ }^{1}$ Instituto de Saúde Coletiva, Universidade Federal da Bahia. R. Basílio da Gama s/n, Canela. 40110 040 Salvador BA Brasil. aambarreto@hotmail.com

\begin{abstract}
The current crisis of capitalism has multiple economic, financial, social, environmental, cultural and political facets. In Brazil, the severity of the crisis is no different, resulting from the exhaustion of the neo-developmentalist model and its inability to resist global crisis. This study compares suicide mortality rates (MR) among employed and unemployed persons in Brazil prior to and during the economic crisis using death records from the period 2011 to 2016. The findings show that in the period 2011 to 2016 the suicide MR fell from 2.66/100,000 to 2.46 among unemployed persons and increased from 5.52/100,000 to $6.89 / 100,000$ in employed persons. Suicide is a complex, multi-causal phenomenon determined by a diverse range of social factors, including strategies that increase worker exploitation. Indeed, being employed can have a greater negative impact on the mental health of workers than being unemployed.
\end{abstract}

Key words Crisis of capitalismo, Economic crisis, Unemployment, Precarious work, Suicide
Resumo A atual crise do capitalismo apresenta um caráter múltiplo: econômico, financeiro, social, ambiental, cultural e político. No Brasil, a gravidade da crise não é diferente, podendo ser explicada como resultado do esgotamento do modelo neodesenvolvimentista, bem como de sua incapacidade de resistir à crise mundial. Este estudo compara o coeficiente de mortalidade (CM) por suicídio de acordo com a situação de ocupação dos brasileiros em periodo anterior e durante a crise econômica. Para isso, foram utilizados os registros de óbitos da população brasileira no período de 2011 a 2016. Os resultados mostram que, entre os desempregados, o CM por suicídio se reduziu de 2,66 óbitos/100 mil, em 2011, para 2,46, em 2016, enquanto, entre os ocupados, aumentou de 5,52 para 6,89 óbitos/100 mil no mesmo período. Compreende-se a complexidade e a multicausalidade da ocorrência do suicídio, sabendo que é um fenômeno socialmente determinado, inclusive pelas estratégias que aumentam a exploração da força de trabalho. Com efeito, estar ocupado, laboralmente, pode ter maior impacto negativo sobre a saúde mental de trabalhadores/as do que estar desempregado.

Palavras-chave Crise do capitalismo, Crise econômica, Desemprego, Precarização do trabalho, Suicídio 


\section{Introdução}

No fim do século XX, o capitalismo vivenciou transformações sócio-históricas que impactaram de modo significativo o mundo do trabalho ${ }^{1,2}$. Após um longo período de crescimento econômico - iniciado no pós-guerra -, os anos 1970 foram marcados pela estagnação de investimentos, com queda persistente na taxa média de lucros, e pela crise do padrão de acumulação taylorista e fordista, explicada por meio das contradições da sua estrutura material de reprodução social, econômica e política, que acabaram minando sua operação contínua de promoção de lucros e expansão econômica ${ }^{1-5}$.

Nesse mesmo período - final da década de 1970 -, surgia no Brasil o Partido dos Trabalhadores (PT), que congregava setores da esquerda e das camadas populares, médias e intelectuais, e expressava uma nova organização dos trabalhadores e trabalhadoras do setor formal ${ }^{6}$. Essa "nova organização" é fruto do deslocamento de parcela considerável da atividade produtiva dos países de capitalismo avançado para áreas localizadas na periferia do sistema capitalista, reduzindo o proletariado industrial naqueles países e expandindo o contingente de trabalhadores/as (sobretudo nos setores de serviços, agroindústria e também na indústria) em vários países no Sul do mundo ${ }^{1}$.

Essa nova divisão internacional do trabalho foi desenhada por meio de medidas que articulavam velhas e novas formas de exploração do trabalho, em resposta aos obstáculos impostos ao processo de acumulação ${ }^{1}$. No contexto brasileiro, a década de 1980 foi marcada pela rearticulação de forças conservadoras, levando a transição democrática a ser acompanhada pela transição econômica ao neoliberalismo ${ }^{6}$. O modelo neoliberal pode ser entendido enquanto uma resposta do capital à sua crise, baseada na articulação de estratégias de extração de mais-valor relativo e absoluto, fundada na superexploração do trabalho'.

Neste cenário, se desenvolve o processo de reestruturação produtiva, desencadeado em meio a condições de exploração particulares e articuladoras de elementos do fordismo com novos mecanismos, próprios das formas de acumulação flexível, transformando a economia, a estrutura social e os padrões de emprego no Brasil ${ }^{1,6}$.

A crise financeira global que eclodiu em 2008 - reflexo tanto das contradições imanentes do capitalismo geral, quanto dos padrões de acumulação construídos a partir de 1970 e desencadeada pelo estouro da bolha imobiliária (no setor dos subprime) - acabou minando muitas das instituições financeiras, além de prejudicar consideravelmente a economia real. Esta crise financeira foi acompanhada de uma recessão nos EUA e em outros países ${ }^{5,7,8}$.

O desemprego provocado pela recessão soma-se, assim, ao desemprego estrutural em escala global (p. 264) que não é um fenômeno recente, se for analisado sob a perspectiva dos países de capitalismo avançado. Com efeito, o desemprego é um desfecho que se tem verificado permanentemente em virtude da crise global do sistema capitalista, tendo surgido como o aspecto necessário e cada vez mais acentuado de uma crise estrutural ${ }^{9,10}$.

Ao desemprego se acompanha a precarização do trabalho, outra estratégia de reprodução do capital que se expressa de várias formas: mercantilização da força de trabalho; padrões de gestão e organização do trabalho levando a condições extremamente inseguras e insalubres inclusive no plano moral com relações baseadas no medo e no abuso de poder (assédio moral e a discriminação criada pela terceirização); constante ameaça de perda de emprego; enfraquecimento da organização sindical, dos movimentos sociais e suas lutas; supressão de direitos sociais anteriormente conquistados ${ }^{11}$.

As condições de trabalho, assim como a falta de trabalho, são parte importante dos determinantes da saúde dos indivíduos e dos grupos sociais. A concepção ampliada da saúde admite que os níveis de saúde da população sofrem determinações sociais, culturais, políticas e econômicas, ultrapassando as dimensões biológicas e ecológicas. Deste modo, é por compreender o caráter socialmente determinado da saúde, que muitos estudos vêm avaliando mudanças no comportamento suicida relacionadas a crises econômicas e a associação destes comportamentos com o desemprego, entre outras consequências das cri$\operatorname{ses}^{12-23}$.

Neste sentido, considerando que o Brasil vive uma crise econômica desde 2014, caracterizada pelo decréscimo do PIB entre os anos de 20142016, com elevação das taxas de desemprego (médias anuais: 2014 - 6,8\%; 2015 - 8,5\%; 2016 - 11,5\%; $2017-12,7 \%)^{24}$, e que o governo federal vem adotando políticas de austeridade fiscal que reduzem a oferta de serviços de proteção social $^{25,26}$, torna-se oportuno estudar tanto o eventual impacto da crise, caracterizada pela elevada taxa de desemprego, quanto o suicídio, importante indicador da situação de saúde/doença de uma população. 
Este estudo tem, portanto, por objetivo estimar e comparar o coeficiente de mortalidade por suicídio de acordo com a situação de ocupação (pessoas registradas com alguma ocupação e pessoas registradas como "desempregadas") na população brasileira em período anterior (20112013) e durante a crise econômica (2014-2016).

\section{Métodos}

Este é um estudo de mortalidade, com base de dados secundários, utilizando-se os registros de óbitos por suicídio da população brasileira para o período de 2011 a 2016. Foram considerados apenas os dados de pessoas com idade igual ou superior a 18 anos.

Trata-se de dados individuados, de domínio público, disponíveis no DATASUS, registrados no Sistema de Informação sobre Mortalidade do Ministério da Saúde - SIM/MS (2011-2016), codificados segundo a Classificação Estatística Internacional de Doenças e Problemas Relacionados à Saúde, na sua décima revisão (CID-10) $)^{27}$. Dados populacionais foram coletados da Pesquisa $\mathrm{Na}-$ cional por Amostra de Domicílios (PNAD) ${ }^{28} \mathrm{e}$ da Pesquisa Nacional por Amostra de Domicílios Contínua (PNAD Contínua) ${ }^{29}$, que se encontram disponíveis publicamente no portal do Instituto Brasileiro de Geografia e Estatística (IBGE).

Levando em conta que o Brasil apresentou uma diminuição do seu Produto Interno Bruto $(\mathrm{PIB})^{30}$ per capita entre os anos 2014-2016 e um aumento da Taxa de Desocupação para o mesmo período, caracterizando assim um estado de recessão econômica, tomamos o ano de 2014 como o início da crise brasileira, para delimitação dos períodos de crise econômica (2014-2016) e de pré-crise (2011-2013).

Embora entendamos que o conceito de classe trabalhadora se refere ao conjunto daqueles que dependem da venda de sua força de trabalho para sobreviver, há de se levar em conta que as estatísticas oficiais sobre trabalho apresentam uma confusão a esse respeito ao classificar a população na força de trabalho como sendo composta por ocupados e desocupados e incorporam à categoria de ocupados todos aqueles que participam da atividade econômica, ou seja, as pessoas que vendem sua força de trabalho (os trabalhadores) e as pessoas que compram essa força de trabalho (os empregadores). Isto significa que os patrões, proprietários dos meios de produção, aparecem junto aos reais trabalhadores ${ }^{31}$.

A variável de desfecho, suicídio, foi definida com base nos códigos da CID-10 registrados na causa básica (<causabas >) compreendendo: lesão autoprovocada intencionalmente (X60 a X84), intoxicação exógena de intenção indeterminada (Y10 a Y19) e sequela de lesões autoprovocadas intencionalmente (Y87.0). Todas as outras causas de mortes foram classificadas como não suicídio. Registre-se que é conhecida a existência de importante subnotificação de suicídio decorrentes de intoxicação exógena devido a possíveis falhas na codificação da causa ${ }^{32,33}$. A variável ocupação (<ocup $>$ ) corresponde à atividade laboral habitual, codificada com a Classificação Brasileira de Ocupações - CBO/2002 $2^{34}$.

A categoria "desempregado" (código 999994 definido pelo DATASUS) da Declaração de Óbito $^{35}$, embora esteja presente no SIM/MS, não é, obviamente, uma ocupação classificada na $\mathrm{CBO} / 2002^{34}$. Logo, como na declaração de óbito registra-se a "ocupação habitual" e não a situação no mercado de trabalho, a categoria "desempregado" (situação de falta de emprego) estaria sendo registrada erroneamente, por não se tratar de uma ocupação ${ }^{37}$.

Outros fatores que podem estar associados aos suicídios foram analisados descritivamente. Essas covariáveis referem-se às seguintes características sociodemográficas e econômicas: sexo, idade, raça/cor autodeclarada (branca, preta, amarela, parda e indígena), situação conjugal (solteiro, casado, viúvo ou divorciado) e escolaridade. Embora existam diversos fatores associados ao risco de suicídio, nem todos foram analisados no presente estudo, visto que utilizamos os dados do SIM, que apresenta um número limitado de variáveis.

A medida de mortalidade foi o coeficiente de mortalidade geral $(\mathrm{CM})$ por suicídio entre pessoas ocupadas e entre pessoas desocupadas por cem mil pessoas-ano, obtido pela divisão do número de óbitos por suicídio pelo número total de ocupados e desocupados no país. O CM por suicídio foi calculado de acordo com a situação de ocupação, considerando outras covariáveis.

Para a análise descritiva, foram apresentadas as frequências absolutas e relativas das características gerais da população e suas distribuições de acordo com o desfecho. O programa Stata 12.0 (Stata Corporation, College Station, USA) foi utilizado para as análises. 


\section{Resultados}

Foram registrados 62.950 óbitos por suicídio no SIM, no período do estudo, dos quais 30.493 ocorreram entre 2011 e 2013 e 32.457 no período de 2014 a 2016. A proporção de óbitos por suicídio foi maior no sexo masculino em ambos os períodos: 79,19\%, no período pré-crise; e $79,91 \%$, no período de crise econômica. Maiores proporções foram registradas entre as pessoas com idade de 25-39 anos no período pré-crise e entre aquelas com 40-59 anos no período de crise econômica (Tabela 1).

A distribuição dos casos por raça/cor revelou maiores proporções entre pessoas brancas em ambos os períodos, embora a maior variação percentual, entre os períodos, tenha sido identifi- cada entre os/as indígenas. Em relação à variável "escolaridade", as pessoas com oito anos ou mais de educação formal tiveram as maiores proporções de suicídio no período pré-crise $(26,05 \%)$ e de crise $(31,30 \%)$ e a maior variação percentual $(20,15 \%)$ entre os dois períodos.

Em todos os grupos de estado civil, os percentuais de suicídio em relação ao total de óbitos praticamente não variaram entre os dois períodos. No que diz respeito à ocupação, a maioria da população do estudo apresentou algum registro de "ocupação habitual", com uma variação percentual positiva de $1,49 \%$ entre os períodos de 2011-2013 e 2014-2016. Poucas pessoas tinham "desempregado" como ocupação registrada, mas foi neste grupo que se observou o maior aumento do número de mortes

Tabela 1. Óbitos por suicídio, de acordo com as características sociodemográficas e econômicas da população de suicídio, e variação percentual do número de óbitos. Brasil, 2011-2016.

\begin{tabular}{|c|c|c|c|c|c|c|}
\hline \multirow{3}{*}{ Variável } & \multicolumn{3}{|c|}{ 2011-2013 } & \multicolumn{3}{|c|}{ 2014-2016 } \\
\hline & \multirow{2}{*}{$\begin{array}{l}\text { Óbitos [n } \\
(\%)]\end{array}$} & \multicolumn{2}{|c|}{ Suicídio n (\%) } & \multirow{2}{*}{$\begin{array}{c}\text { Óbitos [n } \\
(\%)]\end{array}$} & \multicolumn{2}{|c|}{ Suicídio n (\%) } \\
\hline & & Sim & Não & & Sim & Não \\
\hline Total & $\begin{array}{c}3.365 .335 \\
(100)\end{array}$ & $\begin{array}{c}30.493 \\
(0,91)\end{array}$ & $\begin{array}{c}3.334 .842 \\
(99,09)\end{array}$ & $\begin{array}{c}3.613 .041 \\
(100)\end{array}$ & $\begin{array}{l}32.457 \\
(0,90)\end{array}$ & $\begin{array}{c}3.580 .584 \\
(99,10)\end{array}$ \\
\hline \multicolumn{7}{|l|}{ Sexo } \\
\hline Feminino & $\begin{array}{c}1.458 .354 \\
(43,33)\end{array}$ & $\begin{array}{c}6.341 \\
(20,79)\end{array}$ & $\begin{array}{c}1.452 .013 \\
(43,54)\end{array}$ & $\begin{array}{c}1.584 .828 \\
(43,86)\end{array}$ & $\begin{array}{c}6.513 \\
(20,07)\end{array}$ & $\begin{array}{c}1.578 .315 \\
(44,08)\end{array}$ \\
\hline Masculino & $\begin{array}{c}1.905 .929 \\
(56,63)\end{array}$ & $\begin{array}{l}24.147 \\
(79,19)\end{array}$ & $\begin{array}{c}1.881 .782 \\
(56,43)\end{array}$ & $\begin{array}{c}2.026 .787 \\
(56,10)\end{array}$ & $\begin{array}{l}25.938 \\
(79,91)\end{array}$ & $\begin{array}{c}2.000 .849 \\
(55,88)\end{array}$ \\
\hline Ignorado & $1.052(0,03)$ & $5(0,02)$ & $1.047(0,03)$ & $1.426(0,04)$ & $6(0,02)$ & $1.420(0,04)$ \\
\hline \multicolumn{7}{|l|}{ Grupo de idade } \\
\hline $18-24$ anos & $\begin{array}{c}113.607 \\
(3,38)\end{array}$ & $\begin{array}{c}4.273 \\
(14,01)\end{array}$ & $\begin{array}{c}109.334 \\
(3,28)\end{array}$ & $\begin{array}{c}116.428 \\
(3,22)\end{array}$ & $\begin{array}{c}4.341 \\
(13,37)\end{array}$ & $\begin{array}{c}112.087 \\
(3,13)\end{array}$ \\
\hline $25-39$ anos & $\begin{array}{c}276.755 \\
(8,22)\end{array}$ & $\begin{array}{c}10.473 \\
(34,35)\end{array}$ & $\begin{array}{c}266.282 \\
(7,98)\end{array}$ & $\begin{array}{c}276.598 \\
(7,66)\end{array}$ & $\begin{array}{l}10.775 \\
(33,20)\end{array}$ & $\begin{array}{c}265.823 \\
(7,42)\end{array}$ \\
\hline $40-59$ anos & $\begin{array}{l}721.557 \\
(21,44)\end{array}$ & $\begin{array}{c}10.450 \\
(34,27)\end{array}$ & $\begin{array}{l}711.107 \\
(21,32)\end{array}$ & $\begin{array}{c}734.214 \\
(20,32)\end{array}$ & $\begin{array}{l}11.421 \\
(35,19)\end{array}$ & $\begin{array}{l}722.793 \\
(20,19)\end{array}$ \\
\hline 60 anos ou mais & $\begin{array}{c}2.241 .659 \\
(66,61)\end{array}$ & $\begin{array}{c}5.195 \\
(17,04)\end{array}$ & $\begin{array}{c}2.236 .464 \\
(67,06)\end{array}$ & $\begin{array}{c}2.475 .733 \\
(68,52)\end{array}$ & $\begin{array}{c}5.839 \\
(17,99)\end{array}$ & $\begin{array}{c}2.469 .894 \\
(68,98)\end{array}$ \\
\hline \multicolumn{7}{|l|}{ Raça/cor } \\
\hline Branca & $\begin{array}{c}1.766 .773 \\
(52,50)\end{array}$ & $\begin{array}{l}15.146 \\
(49,67)\end{array}$ & $\begin{array}{c}1.751 .627 \\
(52,53)\end{array}$ & $\begin{array}{c}1.866 .609 \\
(51,66)\end{array}$ & $\begin{array}{l}16.243 \\
(50,04)\end{array}$ & $\begin{array}{c}1.850 .366 \\
(51,68)\end{array}$ \\
\hline Preta & $\begin{array}{c}263.665 \\
(7,83)\end{array}$ & $1.768(5,80)$ & $\begin{array}{c}261.897 \\
(7,85)\end{array}$ & $\begin{array}{c}277.245 \\
(7,67)\end{array}$ & $\begin{array}{l}1.735 \\
(5,35)\end{array}$ & $\begin{array}{c}275.510 \\
(7,69)\end{array}$ \\
\hline Amarela & $19.762(0,59)$ & $125(0,41)$ & $\begin{array}{l}19.637 \\
(0,59)\end{array}$ & $\begin{array}{l}20.746 \\
(0,57)\end{array}$ & $139(0,43)$ & $\begin{array}{l}20.607 \\
(0,58)\end{array}$ \\
\hline Parda & $\begin{array}{c}1.124 .704 \\
(33,42)\end{array}$ & $\begin{array}{l}11.998 \\
(39,35)\end{array}$ & $\begin{array}{c}1.112 .706 \\
(33,37)\end{array}$ & $\begin{array}{c}1.284 .961 \\
(35,56)\end{array}$ & $\begin{array}{l}13.137 \\
(40,48)\end{array}$ & $\begin{array}{c}1.271 .824 \\
(35,52)\end{array}$ \\
\hline Indígena & $6.929(0,21)$ & $211(0,69)$ & $6.718(0,20)$ & $7.691(0,21)$ & $246(0,76)$ & $7.445(0,21)$ \\
\hline Ignorado & $\begin{array}{c}183.502 \\
(5,45)\end{array}$ & $1.245(4,08)$ & $\begin{array}{c}182.257 \\
(5,47)\end{array}$ & $\begin{array}{c}155.789 \\
(4,31)\end{array}$ & $957(2,95)$ & $\begin{array}{c}154.832 \\
(4,32)\end{array}$ \\
\hline
\end{tabular}


Tabela 1. Óbitos por suicídio, de acordo com as características sociodemográficas e econômicas da população de suicídio, e variação percentual do número de óbitos. Brasil, 2011-2016.

\begin{tabular}{|c|c|c|c|c|c|c|}
\hline \multirow{3}{*}{ Variável } & \multicolumn{3}{|c|}{ 2011-2013 } & \multicolumn{3}{|c|}{ 2014-2016 } \\
\hline & \multirow{2}{*}{$\begin{array}{c}\text { Óbitos [n } \\
(\%)]\end{array}$} & \multicolumn{2}{|c|}{ Suicídio n (\%) } & \multirow{2}{*}{$\begin{array}{c}\text { Óbitos [n } \\
\text { (\%)] }\end{array}$} & \multicolumn{2}{|c|}{ Suicídio n (\%) } \\
\hline & & Sim & Não & & Sim & Não \\
\hline \multicolumn{7}{|l|}{ Escolaridade } \\
\hline Não estudou & $\begin{array}{c}571.134 \\
(16,97)\end{array}$ & $1.631(5,35)$ & $\begin{array}{c}569.503 \\
(17,08)\end{array}$ & $\begin{array}{c}614.350 \\
(17,00)\end{array}$ & $\begin{array}{l}1.582 \\
(4,87)\end{array}$ & $\begin{array}{c}612.768 \\
(17,11)\end{array}$ \\
\hline $\begin{array}{l}1 \text { a } 3 \text { anos de } \\
\text { educação formal }\end{array}$ & $\begin{array}{l}863.228 \\
(25,65)\end{array}$ & $\begin{array}{c}5.272 \\
(17,29)\end{array}$ & $\begin{array}{c}857.956 \\
(25,73)\end{array}$ & $\begin{array}{l}908.825 \\
(25,15)\end{array}$ & $\begin{array}{c}4.953 \\
(15,26)\end{array}$ & $\begin{array}{l}903.872 \\
(25,24)\end{array}$ \\
\hline $\begin{array}{l}4 \text { a } 7 \text { anos de } \\
\text { educação formal }\end{array}$ & $\begin{array}{l}599.219 \\
(17,81)\end{array}$ & $\begin{array}{c}7.081 \\
(23,22)\end{array}$ & $\begin{array}{l}592.138 \\
(17,76)\end{array}$ & $\begin{array}{l}693.729 \\
(19,20)\end{array}$ & $\begin{array}{c}7.834 \\
(24,14)\end{array}$ & $\begin{array}{l}685.895 \\
(19,16)\end{array}$ \\
\hline $\begin{array}{l}\geq 8 \text { anos de educação } \\
\text { formal }\end{array}$ & $\begin{array}{l}516.276 \\
(15,34)\end{array}$ & $\begin{array}{c}7.944 \\
(26,05)\end{array}$ & $\begin{array}{l}508.332 \\
(15,24)\end{array}$ & $\begin{array}{c}629.814 \\
(17,43)\end{array}$ & $\begin{array}{l}10.160 \\
(31,30)\end{array}$ & $\begin{array}{l}619.654 \\
(17,31)\end{array}$ \\
\hline Ignorado & $\begin{array}{c}815.478 \\
(24,23)\end{array}$ & $\begin{array}{c}8.565 \\
(28,09)\end{array}$ & $\begin{array}{l}806.913 \\
(24,20)\end{array}$ & $\begin{array}{l}766.323 \\
(21,21)\end{array}$ & $\begin{array}{c}7.928 \\
(24,43)\end{array}$ & $\begin{array}{l}758.395 \\
(21,18)\end{array}$ \\
\hline \multicolumn{7}{|l|}{ Estado civil } \\
\hline Solteiro & $\begin{array}{l}840.554 \\
(24,98)\end{array}$ & $\begin{array}{l}14.402 \\
(47,23)\end{array}$ & $\begin{array}{l}826.152 \\
(24,77)\end{array}$ & $\begin{array}{l}907.884 \\
(25,13)\end{array}$ & $\begin{array}{l}15.534 \\
(47,86)\end{array}$ & $\begin{array}{l}892.350 \\
(24,92)\end{array}$ \\
\hline Casado & $\begin{array}{c}1.173 .125 \\
(34,86)\end{array}$ & $\begin{array}{c}8.627 \\
(28,29)\end{array}$ & $\begin{array}{c}1.164 .498 \\
(34,92)\end{array}$ & $\begin{array}{c}1.219 .026 \\
(33,74)\end{array}$ & $\begin{array}{c}9.050 \\
(27,88)\end{array}$ & $\begin{array}{c}1.209 .976 \\
(33,79)\end{array}$ \\
\hline Viúvo & $\begin{array}{l}802.758 \\
(23,85)\end{array}$ & $1.311(4,30)$ & $\begin{array}{l}801.447 \\
(24,03)\end{array}$ & $\begin{array}{l}878.420 \\
(24,31)\end{array}$ & $\begin{array}{l}1.339 \\
(4,13)\end{array}$ & $\begin{array}{l}877.081 \\
(24,50)\end{array}$ \\
\hline $\begin{array}{l}\text { Separado } \\
\text { judicialmente }\end{array}$ & $\begin{array}{c}179.378 \\
(5,33)\end{array}$ & $2.094(6,87)$ & $\begin{array}{c}177.284 \\
(5,32)\end{array}$ & $\begin{array}{c}211.537 \\
(5,85)\end{array}$ & $\begin{array}{l}2.306 \\
(7,10)\end{array}$ & $\begin{array}{c}209.231 \\
(5,84)\end{array}$ \\
\hline Ignorado & $\begin{array}{c}369.520 \\
(10,98)\end{array}$ & $\begin{array}{c}4.059 \\
(13,31)\end{array}$ & $\begin{array}{c}365.461 \\
(10,96)\end{array}$ & $\begin{array}{c}396.174 \\
(10,97)\end{array}$ & $\begin{array}{c}4.228 \\
(13,03)\end{array}$ & $\begin{array}{c}391.946 \\
(10,95)\end{array}$ \\
\hline \multicolumn{7}{|l|}{ Ocupação habitual } \\
\hline Empregado & $\begin{array}{c}1.208 .019 \\
(35,90)\end{array}$ & $\begin{array}{l}16.581 \\
(54,38)\end{array}$ & $\begin{array}{c}1.191 .438 \\
(35,73)\end{array}$ & $\begin{array}{c}1.292 .032 \\
(35,76)\end{array}$ & $\begin{array}{l}17.912 \\
(55,19)\end{array}$ & $\begin{array}{c}1.274 .120 \\
(35,58)\end{array}$ \\
\hline Desempregado & $22.437(0,67)$ & $457(1,50)$ & $\begin{array}{r}21.980 \\
(0,66) \\
\end{array}$ & $\begin{array}{c}35.224 \\
(0,97)\end{array}$ & $728(2,24)$ & $\begin{array}{c}34.496 \\
(0,96) \\
\end{array}$ \\
\hline
\end{tabular}

Fonte: SIM.

por suicídio, que passou de 457 óbitos em 20112013 para 728 óbitos em 2014-2016 (Tabela 1). Apesar desse aumento, o CM por suicídio foi maior entre pessoas registradas com alguma ocupação do que entre as pessoas cuja ocupação foi registrada como "desempregado" em todos os anos do estudo.

Ao longo do período de 2011-2013, observou-se uma variação percentual positiva do CM por suicídio de $4,3 \%$ entre pessoas registradas com alguma ocupação, enquanto entre as pessoas registradas como "desempregadas" ocorreu uma variação percentual positiva de $51 \%$ no CM por suicídio. Para o período de 2014-2016, o CM por suicídio de pessoas registradas com alguma ocupação aumentou $25,0 \%$, enquanto para pessoas registradas como "desempregadas" diminuiu de $43 \%$. Entre as pessoas registradas como "desempregadas", o maior valor do CM por sui- cídio ocorreu em 2014 (CM 4,31/100.000), enquanto para as pessoas registradas com alguma ocupação o maior valor ocorreu em 2016 (CM 6,89/100.000) (Gráfico 1).

Em média, o CM por suicídio no sexo masculino foi maior entre pessoas registradas com alguma ocupação do que entre as pessoas registradas como desempregadas. Entre as pessoas registradas como "desempregadas", o maior valor do CM por suicídio ocorreu em 2014 (CM 11,46/100 mil), enquanto para as pessoas registradas com alguma ocupação o maior valor ocorreu em 2016 (CM 10,39/100 mil). Ao longo do período pré-crise, observou-se um aumento de $4,8 \%$ no CM por suicídio entre homens registrados com alguma ocupação. Para aqueles registrados como "desempregados", o CM por suicídio aumentou $54,5 \%$. No período de crise, houve um aumento de $21,6 \%$ no CM por suicídio de homens registrados com algu- 
ma ocupação, enquanto entre os “desempregados" o CM por suicídio diminuiu 62,3\% (Gráfico 2).

O CM por suicídio de mulheres registradas com alguma ocupação foi maior em relação ao das mulheres registradas como desempregadas, em todos os anos estudados (Gráfico 3). No início do período, em 2011, o CM por suicídio foi $1,77 / 100$ mil entre mulheres com alguma ocupação e 0,63/100 mil entre mulheres desempregadas. Ao longo do período de crise, houve um aumento de 30,3\% no CM por suicídio de mulheres registradas com alguma ocupação, enquanto en- tre as "desempregadas" o CM por suicídio diminuiu 7,1\% (Gráfico 3).

Quanto ao registro da ocupação, a maior parte dos casos era representada por trabalhadores na exploração agropecuária (2011-2013: 23,17\%; 2014-2016: 20,97\%), seguidos de trabalhadores da indústria extrativa e da construção civil (2011-2013: 15,06\%; 2014-2016: 14,81\%), trabalhadores dos serviços (2011-2013: 13,43\%; 2014-2016: 13,47\%) e trabalhadores de funções transversais (2011-2013: 6,22\%; 2014-2016: 6,40\%), nesta ordem (Tabela 2).

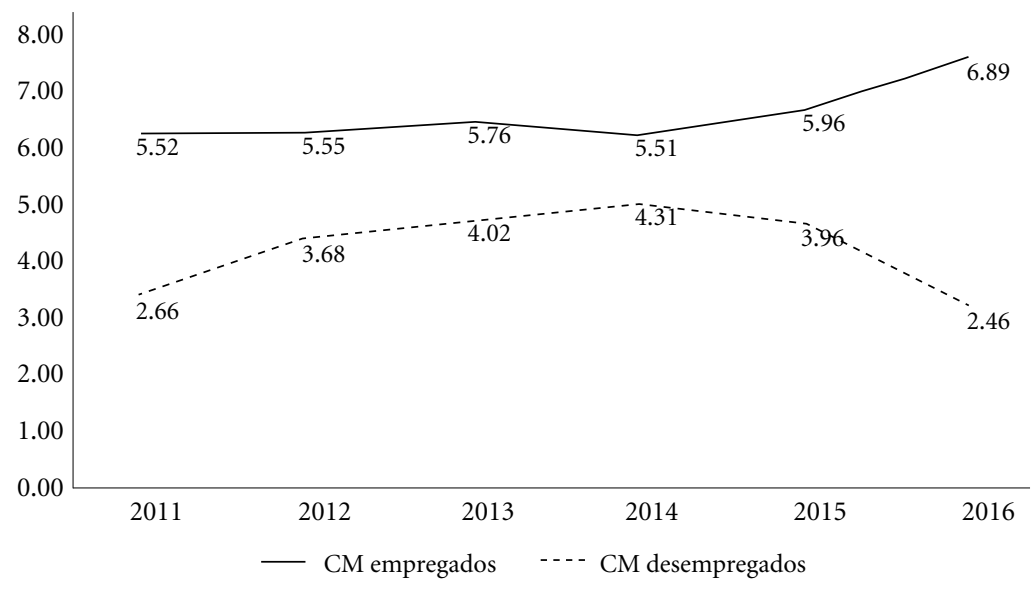

Gráfico 1. Coeficiente de mortalidade de suicídio, segundo situação de ocupação. Brasil, 2011-2016.

Fonte: SIM e IBGE.

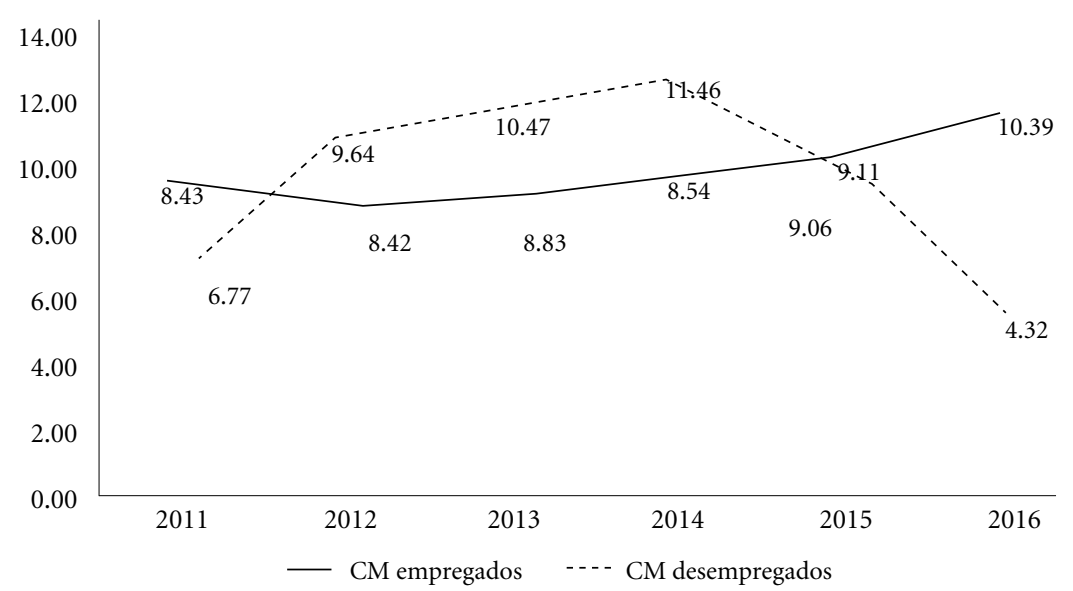

Gráfico 2. Coeficiente de mortalidade por suicídio em pessoas registradas como "desempregadas" e com alguma ocupação, segundo sexo masculino. Brasil, 2011-2016. 


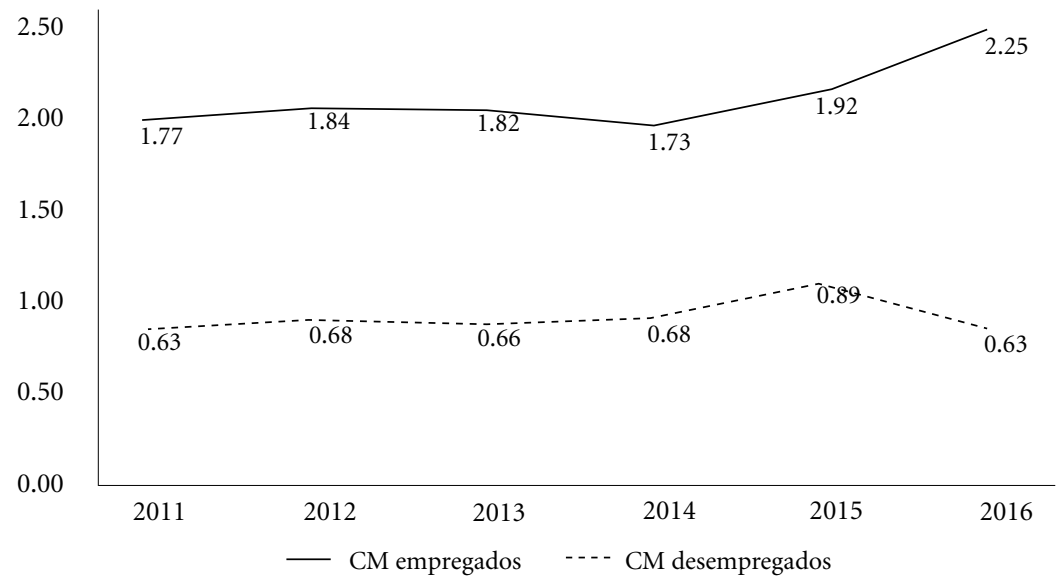

Gráfico 3. Coeficiente de mortalidade por suicídio em pessoas registradas como "desempregadas" e com alguma ocupação, segundo sexo feminino. Brasil, 2011-2016.

Fonte: SIM e IBGE.

\section{Discussão}

Neste estudo, os maiores CM por suicídio foram encontrados entre os homens. Em outros países - sobretudo, europeus - estudos realizados, notadamente após 2008, identificaram que o suicídio é mais frequente entre os homens ${ }^{38-40}$. No Brasil, esse mesmo resultado foi encontrado por outros estudos ${ }^{41,42}$. A maior frequência de suicídio de homens pode estar associada a fatores como impulsividade, agressividade, uso de substâncias psicoativas ${ }^{43-45}$ e uso de métodos mais letais na tentativa de suicídio ${ }^{46,47}$.

Observamos uma variação percentual negativa do CM por suicídio entre as pessoas registradas como "desempregadas" quando comparada às pessoas registradas com alguma ocupação, no período de crise. Asevedo et al. examinaram a relação entre taxas de suicídio e indicadores econômicos (PIB per capita e taxas de desemprego) em grandes centros urbanos brasileiros (entre 2006 e 2015) e sugeriram que a redução das taxas de desemprego estava correlacionada com maiores taxas de suicídio ${ }^{48}$. Bando et al., em 2010, encontraram maiores taxas de suicídio nas regiões do Brasil com alta renda per capita e menores taxas de desemprego, sugerindo que o suicídio é mais comum em regiões com maior qualidade de vida $^{41}$. Tais resultados coincidem com os nossos, ainda que tenham analisado dados agregados ${ }^{41,48}$ (sendo uma limitação, pois não permite a inferência do efeito do desemprego no nível do indivíduo).
Machado et al., por sua vez, mostraram que a desigualdade de renda é um determinante do suicídio no Brasil. Identificam, entre 2000 e 2011, um menor aumento da taxa de suicídio, comparado a períodos anteriores, atribuindo esse efeito à redução da desigualdade social, à redução da porcentagem de indivíduos que não concluíram os estudos básicos e ao aumento nas rendas ${ }^{49}$.

Estudos que analisaram a relação entre indicadores socioeconômicos e suicídio sugerem que o desemprego decorrente da crise econômica e de medidas de austeridade fiscal contribui para o aumento das taxas de suicídio ${ }^{13,15,16,20,25,38,39,50,51}$. Ao contrário, os resultados de nosso estudo indicam que os brasileiros "ocupados" estão mais expostos ao risco de morrer por suicídio do que os registrados como "desempregados".

O maior risco de morrer por suicídio entre as pessoas que registradas com alguma ocupação, quando comparado às pessoas registradas como "desempregadas" pode estar relacionado às mudanças ocorridas no mundo do trabalho nas últimas décadas. $\mathrm{O}$ sofrimento psíquico associado ao trabalho está ligado diretamente à lógica destrutiva do capital, que não reconhece nenhuma barreira para a precarização do trabalho, explorando a força de trabalho ao extremo, ao tempo que abrevia seu tempo de uso e coloca os trabalhadores num patamar de descartabilidade (uma vez que muitos acabam por ficar incapacitados de forma definitiva para o trabalho $)^{52}$.

A disseminação global dos processos de reorganização do trabalho e da produção e, de 
Tabela 2. Distribuição dos registros da ocupação em casos de suicídio, no Sistema de Informação sobre Mortalidade, por consistência. Brasil, 2011-2013, 2014-2016.

\begin{tabular}{|c|c|c|c|c|c|c|c|}
\hline \multicolumn{4}{|c|}{ 2011-2013 } & \multicolumn{4}{|c|}{ 2014-2016 } \\
\hline & Registro de ocupação & $\begin{array}{c}N= \\
16.581\end{array}$ & $\%$ & & Registro de ocupação & $\begin{array}{c}\mathrm{N}= \\
17.912\end{array}$ & $\%$ \\
\hline $1^{\circ}$ & $\begin{array}{l}\text { Trabalhadores na exploração } \\
\text { agropecuária }\end{array}$ & 3.841 & 23,17 & 10 & $\begin{array}{l}\text { Trabalhadores na exploração } \\
\text { agropecuária }\end{array}$ & 3.756 & 20,97 \\
\hline $2^{\circ}$ & $\begin{array}{l}\text { Trabalhadores da indústria } \\
\text { extrativa e da construção civil }\end{array}$ & 2.497 & 15,06 & $2^{\circ}$ & $\begin{array}{l}\text { Trabalhadores da indústria } \\
\text { extrativa e da construção civil }\end{array}$ & 2.652 & 14,81 \\
\hline $3^{\circ}$ & Trabalhadores dos serviços & 2.226 & 13,43 & $3^{\circ}$ & Trabalhadores dos serviços & 2.413 & 13,47 \\
\hline $4^{\circ}$ & $\begin{array}{l}\text { Trabalhadores de funções } \\
\text { transversais }\end{array}$ & 1.031 & 6,22 & $4^{\circ}$ & $\begin{array}{l}\text { Trabalhadores de funções } \\
\text { transversais }\end{array}$ & 1.147 & 6,40 \\
\hline $5^{\circ}$ & $\begin{array}{l}\text { Produtores na exploração } \\
\text { agropecuária }\end{array}$ & 707 & 4,26 & $5^{\circ}$ & $\begin{array}{l}\text { Técnicos de nível médio nas } \\
\text { ciências administrativas }\end{array}$ & 824 & 4,60 \\
\hline $6^{\circ}$ & Gerentes & 702 & 4,23 & $6^{\circ}$ & $\begin{array}{l}\text { Produtores na exploração } \\
\text { agropecuária }\end{array}$ & 784 & 4,38 \\
\hline $7^{\mathbf{o}}$ & $\begin{array}{l}\text { Técnicos de nível médio nas } \\
\text { ciências administrativas }\end{array}$ & 612 & 3,69 & $7^{0}$ & Gerentes & 710 & 3,96 \\
\hline $8^{\circ}$ & $\begin{array}{l}\text { Vendedores e prestadores de } \\
\text { serviços do comércio }\end{array}$ & 610 & 3,68 & $8^{\circ}$ & $\begin{array}{l}\text { Vendedores e prestadores de } \\
\text { serviços do comércio }\end{array}$ & 691 & 3,86 \\
\hline $9^{\circ}$ & $\begin{array}{l}\text { Trabalhadores da transformação de } \\
\text { metais e de compósitos }\end{array}$ & 440 & 2,65 & $9^{\circ}$ & $\begin{array}{l}\text { Trabalhadores da transformação de } \\
\text { metais e de compósitos }\end{array}$ & 482 & 2,69 \\
\hline $10^{\circ}$ & Escriturários & 387 & 2,33 & $10^{\circ}$ & Escriturários & 387 & 2,16 \\
\hline $11^{\circ}$ & $\begin{array}{l}\text { Trabalhadores em serviços de } \\
\text { reparação e manutenção mecânica }\end{array}$ & 262 & 1,58 & $11^{\circ}$ & $\begin{array}{l}\text { Profissionais das ciências } \\
\text { biológicas, da saúde e afins }\end{array}$ & 302 & 1,69 \\
\hline $12^{\circ}$ & $\begin{array}{l}\text { Profissionais das ciências } \\
\text { biológicas, da saúde e afins }\end{array}$ & 240 & 1,45 & $12^{\circ}$ & $\begin{array}{l}\text { Profissionais das ciências sociais e } \\
\text { humanas }\end{array}$ & 284 & 1,59 \\
\hline $13^{\circ}$ & $\begin{array}{l}\text { Profissionais das ciências sociais e } \\
\text { humanas }\end{array}$ & 240 & 1,45 & $13^{\circ}$ & $\begin{array}{l}\text { Trabalhadores em serviços de } \\
\text { reparação e manutenção mecânica }\end{array}$ & 265 & 1,48 \\
\hline $14^{\circ}$ & $\begin{array}{l}\text { Trabalhadores nas indústrias têxtil, } \\
\text { do curtimento, do vestuário e das } \\
\text { artes gráficas }\end{array}$ & 215 & 1,30 & $14^{\circ}$ & $\begin{array}{l}\text { Trabalhadores nas indústrias têxtil, } \\
\text { do curtimento, do vestuário e das } \\
\text { artes gráficas }\end{array}$ & 264 & 1,47 \\
\hline $15^{\circ}$ & Profissionais do ensino & 201 & 1,21 & $15^{\circ}$ & $\begin{array}{l}\text { Técnicos de nível médio das ciências } \\
\text { físicas, químicas, engenharia e afins }\end{array}$ & 217 & 1,21 \\
\hline
\end{tabular}

maneira articulada, a expansão das diferentes formas de precarização do trabalho, tais como a expansão da terceirização, o assédio moral, a gestão por metas e a redução dos direitos trabalhistas se relacionam com a incidência de sofrimento mental entre trabalhadores e trabalhadoras ${ }^{52}$ e, possivelmente, com o risco aumentado de morte por suicídio.

Vale destacar duas convergências com outros estudos: o aumento no CM por suicídio no período de crise $\mathrm{e}^{14,20,38,53-57}$ e o maior registro de suicídio entre trabalhadores/as na exploração agropecuária $^{58-60}$. Meyer et al., em estudo realizado no Rio de Janeiro, mostraram que a mortalidade por suicídio foi maior entre os residentes das áreas rurais, principalmente os trabalhadores agrícolas residentes nessas áreas, sugerindo que estes trabalhadores, por viverem em áreas com o uso mais intenso de pesticidas, também estavam em maior risco de mortalidade por suicídio, o que pode ser explicado por um aumento no risco de depressão e tentativas de suicídio como consequência da exposição contínua a esses compostos neurotóxicos ${ }^{60}$.

Há divergência, contudo, entre nossos achados e os de estudos que viram maior risco de suicídio entre empresários e funcionários de alta patente, no período de crise econômica ${ }^{54,61}$.

A diferença entre os nossos achados e os de outros estudos, relativamente ao desemprego, 
Tabela 2. Distribuição dos registros da ocupação em casos de suicídio, no Sistema de Informação sobre Mortalidade, por consistência. Brasil, 2011-2013, 2014-2016.

\begin{tabular}{|c|c|c|c|c|c|c|c|}
\hline \multicolumn{4}{|c|}{ 2011-2013 } & \multicolumn{4}{|c|}{ 2014-2016 } \\
\hline & Registro de ocupação & $\begin{array}{l}N= \\
16.581\end{array}$ & $\%$ & & Registro de ocupação & $\begin{array}{c}\mathrm{N}= \\
17.912\end{array}$ & $\%$ \\
\hline $16^{\circ}$ & $\begin{array}{l}\text { Técnicos de nível médio das ciências } \\
\text { físicas, químicas, engenharia e afins }\end{array}$ & 181 & 1,09 & $16^{\circ}$ & Profissionais do ensino & 215 & 1,20 \\
\hline $17 \mathrm{o}$ & $\begin{array}{l}\text { Trabalhadores de atendimento ao } \\
\text { público }\end{array}$ & 178 & 1,07 & $17^{\circ}$ & $\begin{array}{l}\text { Trabalhadores de atendimento ao } \\
\text { público }\end{array}$ & 196 & 1,09 \\
\hline $18^{\circ}$ & Policiais militares & 172 & 1,04 & $18^{\circ}$ & Comunicadores, artistas e religiosos & 188 & 1,05 \\
\hline $19^{\circ}$ & $\begin{array}{l}\text { Comunicadores, artistas e } \\
\text { religiosos }\end{array}$ & 157 & 0,95 & 190 & $\begin{array}{l}\text { Trabalhadores da fabricação de } \\
\text { alimentos, bebidas e fumo }\end{array}$ & 187 & 1,04 \\
\hline $20^{\circ}$ & $\begin{array}{l}\text { Técnicos de nível médio das } \\
\text { ciências biológicas, bioquímicas, } \\
\text { da saúde e afins }\end{array}$ & 154 & 0,93 & $20^{\circ}$ & $\begin{array}{l}\text { Profissionais das ciências exatas, } \\
\text { físicas e da engenharia }\end{array}$ & 186 & 1,04 \\
\hline $21^{\circ}$ & $\begin{array}{l}\text { Trabalhadores da fabricação de } \\
\text { alimentos, bebidas e fumo }\end{array}$ & 142 & 0,86 & $21^{\circ}$ & $\begin{array}{l}\text { Técnicos de nível médio das ciências } \\
\text { biológicas, bioquímicas, da saúde } \\
\text { e afins }\end{array}$ & 180 & 1,00 \\
\hline $22^{\circ}$ & $\begin{array}{l}\text { Trabalhadores das indústrias de } \\
\text { madeira e do mobiliário }\end{array}$ & 141 & 0,85 & $22^{\circ}$ & Profissionais das ciências jurídicas & 178 & 0,99 \\
\hline $23^{\circ}$ & $\begin{array}{l}\text { Outros trabalhadores da conservação, } \\
\text { manutenção e reparação }\end{array}$ & 140 & 0,84 & $23^{\circ}$ & Policiais militares & 169 & 0,94 \\
\hline $24^{\circ}$ & Profissionais das ciências jurídicas & 137 & 0,83 & $24^{\circ}$ & $\begin{array}{l}\text { Trabalhadores das indústrias de } \\
\text { madeira e do mobiliário }\end{array}$ & 169 & 0,94 \\
\hline $25^{\circ}$ & $\begin{array}{l}\text { Profissionais das ciências exatas, } \\
\text { físicas e da engenharia }\end{array}$ & 127 & 0,77 & $25^{\circ}$ & Pescadores e extrativistas florestais & 146 & 0,82 \\
\hline $26^{\circ}$ & Pescadores e extrativistas florestais & 115 & 0,69 & $26^{\circ}$ & $\begin{array}{l}\text { Outros trabalhadores da conservação, } \\
\text { manutenção e reparação }\end{array}$ & 134 & 0,75 \\
\hline $27^{\circ}$ & $\begin{array}{l}\text { Operadores de produção, captação, } \\
\text { tratamento e distribuição (energia, } \\
\text { água e utilidades) }\end{array}$ & 104 & 0,63 & $27^{\circ}$ & $\begin{array}{l}\text { Membros superiores e dirigentes } \\
\text { do poder público }\end{array}$ & 130 & 0,73 \\
\hline $28^{\circ}$ & $\begin{array}{l}\text { Membros superiores e dirigentes } \\
\text { do poder público }\end{array}$ & 98 & 0,59 & $28^{\circ}$ & $\begin{array}{l}\text { Operadores de produção, captação, } \\
\text { tratamento e distribuição (energia, } \\
\text { água e utilidades) }\end{array}$ & 122 & 0,68 \\
\hline 290 & $\begin{array}{l}\text { Trabalhadores de instalações } \\
\text { siderúrgicas e de materiais de } \\
\text { construção }\end{array}$ & 84 & 0,51 & 290 & $\begin{array}{l}\text { Trabalhadores de instalações } \\
\text { siderúrgicas e de materiais de } \\
\text { construção }\end{array}$ & 76 & 0,42 \\
\hline $30^{\circ}$ & Professores leigos e de nível médio & 69 & 0,42 & $30^{\circ}$ & Professores leigos e de nível médio & 69 & 0,39 \\
\hline
\end{tabular}

pode estar relacionada aos diferentes métodos adotados, destacando-se o fato dos registros do SIM, analisados neste trabalho, se referirem à ocupação habitual e não à inserção no mercado de trabalho.

Compreendemos a complexidade e a multicausalidade do suicídio, como um fenômeno socialmente determinado, inclusive por estratégias de superexploração da força de trabalho ${ }^{1}$ (e.g.: formas de avaliação de desempenho no local de trabalho; aumento da intensidade e ritmo de produção; assédio moral etc.), o que impacta negativamente a saúde de trabalhadores/as e determina materialmente o sofrimento psíquico.

Por fim, é preciso registrar duas limitações metodológicas do estudo. A primeira se refere à não -inclusão, na pesquisa empírica, de diversos fatores associados ao risco de suicídio, bem documentados na literatura especializada. A segunda limitação decorre da baixa completitude de parte dos dados utilizados (e.g., raça/cor, escolaridade, estado civil e situação de trabalho), da já mencionada inconsistência da variável "ocupação habitual” como medida da inserção no mercado de trabalho. 
Tabela 2. Distribuição dos registros da ocupação em casos de suicídio, no Sistema de Informação sobre Mortalidade, por consistência. Brasil, 2011-2013, 2014-2016.

\begin{tabular}{|c|c|c|c|c|c|c|c|}
\hline \multicolumn{4}{|c|}{ 2011-2013 } & \multicolumn{4}{|c|}{ 2014-2016 } \\
\hline & Registro de ocupação & $\begin{array}{c}N= \\
16.581\end{array}$ & $\%$ & & Registro de ocupação & $\begin{array}{c}\mathrm{N}= \\
17.912\end{array}$ & $\%$ \\
\hline $31^{\circ}$ & $\begin{array}{l}\text { Dirigentes de empresas e } \\
\text { organizações (exceto de interesse } \\
\text { público) }\end{array}$ & 68 & 0,41 & $31^{\circ}$ & $\begin{array}{l}\text { Dirigentes de empresas e } \\
\text { organizações (exceto de interesse } \\
\text { público) }\end{array}$ & 64 & 0,36 \\
\hline $32^{\circ}$ & $\begin{array}{l}\text { Joalheiros, vidreiros, ceramistas e } \\
\text { afins }\end{array}$ & 44 & 0,27 & $32^{\circ}$ & $\begin{array}{l}\text { Trabalhadores da mecanização } \\
\text { agropecuária e florestal }\end{array}$ & 61 & 0,34 \\
\hline $33^{\circ}$ & $\begin{array}{l}\text { Trabalhadores da fabricação e } \\
\text { instalação eletroeletrônica }\end{array}$ & 40 & 0,24 & $33^{\circ}$ & $\begin{array}{l}\text { Técnicos em nível médio dos } \\
\text { serviços culturais, das comunicações } \\
\text { e dos desportos }\end{array}$ & 43 & 0,24 \\
\hline $34^{\circ}$ & $\begin{array}{l}\text { Trabalhadores da mecanização } \\
\text { agropecuária e florestal }\end{array}$ & 40 & 0,24 & $34^{\circ}$ & $\begin{array}{l}\text { Joalheiros, vidreiros, ceramistas e } \\
\text { afins }\end{array}$ & 42 & 0,23 \\
\hline $35^{\circ}$ & $\begin{array}{l}\text { Técnicos em nível médio dos } \\
\text { serviços culturais, das comunicações } \\
\text { e dos desportos }\end{array}$ & 37 & 0,22 & $35^{\circ}$ & Polimantenedores & 37 & 0,21 \\
\hline $36^{\circ}$ & Membros das forças armadas & 29 & 0,17 & $36^{\circ}$ & $\begin{array}{l}\text { Técnicos de nível médio em } \\
\text { serviços de transportes }\end{array}$ & 28 & 0,16 \\
\hline $37 \mathrm{o}$ & Polimantenedores & 27 & 0,16 & $37^{\circ}$ & $\begin{array}{l}\text { Trabalhadores em indústrias de } \\
\text { processos contínuos e outras } \\
\text { indústrias }\end{array}$ & 24 & 0,13 \\
\hline $38^{\circ}$ & $\begin{array}{l}\text { Trabalhadores em indústrias de } \\
\text { processos contínuos e outras } \\
\text { indústrias }\end{array}$ & 20 & 0,12 & $38^{\circ}$ & $\begin{array}{l}\text { Trabalhadores da fabricação e } \\
\text { instalação eletroeletrônica }\end{array}$ & 23 & 0,13 \\
\hline 390 & Bombeiros militares & 18 & 0,11 & $39^{\circ}$ & Membros das forças armadas & 18 & 0,10 \\
\hline $40^{\circ}$ & $\begin{array}{l}\text { Técnicos de nível médio em } \\
\text { serviços de transportes }\end{array}$ & 15 & 0,09 & $40^{\circ}$ & Bombeiros militares & 16 & 0,09 \\
\hline $41^{\circ}$ & Técnicos polivalentes & 11 & 0,07 & $41^{\circ}$ & Outros técnicos de nível médio & 11 & 0,06 \\
\hline $42^{\circ}$ & Outros técnicos de nível médio & 8 & 0,05 & $42^{\circ}$ & $\begin{array}{l}\text { Pesquisadores e profissionais } \\
\text { policientíficos }\end{array}$ & 11 & 0,06 \\
\hline $43^{\circ}$ & $\begin{array}{l}\text { Montadores de aparelhos e } \\
\text { instrumentos de precisão e } \\
\text { musicais }\end{array}$ & 6 & 0,04 & $43^{\circ}$ & Técnicos polivalentes & 5 & 0,03 \\
\hline $44^{\circ}$ & $\begin{array}{l}\text { Pesquisadores e profissionais } \\
\text { policientíficos }\end{array}$ & 4 & 0,02 & $44^{\circ}$ & $\begin{array}{l}\text { Trabalhadores de instalações e } \\
\text { máquinas de fabricação de celulose } \\
\text { e papel }\end{array}$ & 3 & 0,02 \\
\hline $45^{\circ}$ & $\begin{array}{l}\text { Diretores e gerentes em empresa } \\
\text { de serviços de saúde, da educação, } \\
\text { ou de serviços culturais, sociais ou } \\
\text { pessoais }\end{array}$ & 3 & 0,02 & $45^{\circ}$ & $\begin{array}{l}\text { Montadores de aparelhos e } \\
\text { instrumentos de precisão e } \\
\text { musicais }\end{array}$ & 2 & 0,01 \\
\hline $46^{\circ}$ & $\begin{array}{l}\text { Trabalhadores de instalações e } \\
\text { máquinas de fabricação de celulose } \\
\text { e papel }\end{array}$ & 1 & 0,01 & $46^{\circ}$ & $\begin{array}{l}\text { Diretores e gerentes em empresa } \\
\text { de serviços de saúde, da educação, } \\
\text { ou de serviços culturais, sociais ou } \\
\text { pessoais }\end{array}$ & 1 & 0,01 \\
\hline
\end{tabular}

Fonte: SIM.

\section{Conclusões}

Este estudo discute as relações entre desemprego (como "ocupação habitual", de acordo com o SIM) e suicídio, comparando duas conjunturas econô- micas: pré-crise (2011-2013) e crise (2014-2016). Não se observou aumento do CM por suicídio entre as pessoas registradas como "desempregadas", diferentemente do que ocorreu em outros países, notadamente na Grécia após 2008. Esse resultado, 
contudo, não significa que o desemprego seja fator protetor contra o suicídio. Pode estar sugerindo que as condições de trabalho impostas pela nova morfologia do trabalho (e.g., a redução dos direitos trabalhistas, a informalidade e a precarização das condições de vida em geral) expõem as pessoas registradas em alguma ocupação a um risco de morrer por suicídio ainda maior do que o desemprego.

As consequências do sistema de metabolismo societal do capital sobre o mundo do trabalho apresentam efeitos no modo de vida das pessoas, que se materializam na relação direta entre trabalho e saúde, gerando o adoecimento físico e/ou mental de trabalhadores e trabalhadoras ao redor do mundo.

Em vista de tal cenário, é insuficiente pensar em estratégias de mitigação da crise do capitalismo, que minimizem seus impactos sobre a saúde das pessoas. Para que esta realidade seja transformada, coloca-se o desafio de gestar uma organização societal que elimine a superexploração do trabalho, num projeto que vislumbre a desmontagem do processo de acumulação capitalista, por meio de medidas que recusem a lógica do capital e contribuam para a emancipação da humanidade e a sustentabilidade planetária.

\section{Colaboradores}

AAM Barreto idealizou o estudo, participou da coleta e análise de dados, da interpretação e discussão dos resultados, escreveu o manuscrito, tendo contribuído como primeiro autor neste manuscrito. LEPF Souza idealizou o estudo, participou da interpretação e discussão dos resultados, colaborou na escrita do artigo e revisão crítica deste manuscrito. 


\section{Referências}

1. Antunes R. O privilégio da servidão: o novo proletariado de serviços na era digital. $1^{\text {a }}$ ed. São Paulo: Boitempo; 2018.

2. Tauss A. Contextualizing the current crisis: Post-Fordism, neoliberal restructuring, and financialization. Colomb Int 2012; (76):51-79.

3. Fine B, Saad-Filho A. Thirteen Things You Need to Know About Neoliberalism. Crit Sociol 2017; 43(45):685-706.

4. Grespan J. A crise de sobreacumulação. Crítica Marx 2009; (29):11-17.

5. Kotz DM. The financial and economic crisis of 2008: a systemic crisis of neoliberal capitalism. Rev Radic Polit Econ 2009; 41(3):305-317.

6. Saad Filho A. Avanços, contradições e limites dos governos petistas. Crítica Marx 2016; (42):171-177.

7. Carcanholo RA. A atual crise do capitalismo. Crítica Marx 2009; (29):49-55.

8. Filgueiras L. A crise geral do capitalismo: possibilidades e limites de sua superação. Crítica Marx 2010; (30):21-27.

9. Antunes R. Adeus ao trabalho? ensaio sobre as metamorfoses e a centralidade do mundo do trabalho. $16^{\mathrm{a}} \mathrm{ed}$. São Paulo: Cortez; 2015.

10. Mészáros I. Desemprego e "precarização flexível." In: Mészáros I. O desafio e o fardo do tempo do tempo histórico. São Paulo: Boitempo; 2007. p. 400.

11. Druck G. A terceirização sem limites: mais precarização e riscos de morte aos trabalhadores. Cad Saude Publica 2016;32(6):e00146315.

12. Alameda-Palacios J, Ruiz-Ramos M, García-Robredo B. Suicide, antidepressant prescription and unemployment in Andalusia (Spain). Gac Sanit 2014; 28(4):309-312.

13. Antonakakis N, Collins A. The impact of fiscal austerity on suicide: on the empirics of a modern Greek tragedy. Soc Sci Med 2014; 112:39-50.

14. Barr B, Taylor-Robinson D, Scott-Samuel A, McKee M, Stuckler D. Suicides associated with the 2008-10 economic recession in England: Time trend analysis. BMJ 2012; 345(7873):e5142.

15. Coope C, Gunnell D, Hollingworth W, Hawton K, Kapur N, Fearn V, Wells C, Metcalfe C. Suicide and the 2008 economic recession: Who is most at risk? Trends in suicide rates in England and Wales 2001-2011. Soc Sci Med 2014; 117:76-85.

16. Córdoba-Doña JA, San Sebastián M, Escolar-Pujolar A, Martínez-Faure JE, Gustafsson PE. Economic crisis and suicidal behaviour: The role of unemployment, sex and age in Andalusia, Southern Spain. Int J Equity Health 2014; 13(1).

17. Economou M, Madianos M, Peppou LE, Theleritis C, Patelakis A, Stefanis C. Suicidal ideation and reported suicide attempts in Greece during the economic crisis. World Psych 2013; 12(1):53-59.

18. Garcy AM, Vagerö D. Unemployment and suicide during and after a deep recession: A longitudinal study of 3.4 million swedish men and women. Am J Public Health 2013; 103(6):1031-1038.

19. Hong J, Knapp M, Mcguire A. Income-related inequalities in the prevalence of depression and suicidal behaviour: A 10-year trend following economic crisis. World Psych 2011; 10(1):40-44.
20. Lopez Bernal JA, Gasparrini A, Artundo CM, McKee M. The effect of the late 2000s financial crisis on suicides in Spain: An interrupted time-series analysis. Eur J Public Health 2013; 23(5):732-736.

21. Pompili M, Vichi M, Innamorati M, Lester D, Yang B, De Leo D, Girardi P. Suicide in Italy during a time of economic recession: Some recent data related to age and gender based on a nationwide register study. Heal Soc Care Community 2014; 22(4):361-367.

22. Reeves A, McKee M, Stuckler D. Economic suicides in the Great Recession in Europe and North America. $\mathrm{Br}$ J Psychiatry 2014; 205(3):246-247.

23. Reeves A, Stuckler D, McKee M, Gunnell D, Chang $\mathrm{S}-\mathrm{S}$, Basu S. Increase in state suicide rates in the USA during economic recession. Lancet 2012; 380(9856): 1813-1814.

24. Instituto Brasileiro de Geografia e Estatística (IBGE). Coordenação de População e Indicadores Sociais. Síntese de indicadores sociais: uma análise das condições de vida da população brasileira: 2018/IBGE [Internet]. Rio de Janeiro: IBGE; 2018 [acessado 2019 jul 27]. Disponível em: https://biblioteca.ibge.gov.br/visualizacao/livros/liv101629.pdf

25. 25. Vieira FS. Crise econômica, austeridade fiscal e saúde: que lições podem ser aprendidas? [Internet]. Brasília: Instituto de Pesquisa Econômica Aplicada; 2016. [acessado 2019 jul 27]. Disponível em: http:// www.ipea.gov.br/portal/images/stories/PDFs/nota tecnica/160822_nt_26_disoc.pdf

26. Mathias M. A crise por trás da nova PNAB. Rev Poli Saude Educ Trab [Internet]. Rio de Janeiro: EPSJV/ Fiocruz; 2017;(53):6-13. [acessado 2019 jul 27]. Disponível em: http://www.epsjv.fiocruz.br/sites/default/ files/poliweb53.pdf

27. Centro Colaborador da OMS para a Classificação de Doenças em Português (CBCD). Classificação Estatística Internacional de Doenças e Problemas Relacionados à Saúde - CID-10 [Internet]. [acessado 2018 set 19]. Disponível em: http://www.datasus.gov.br/cid10/ V2008/cid10.htm

28. Instituto Brasileiro de Geografia e Estatística (IBGE). Sistema IBGE de Recuperação Automática (SIDRA). Pesquisa Nacional por Amostra de Domicílios [Internet]. Rio de Janeiro: IBGE; 2015 [acessado 2019 jul 27]. Disponível em: https://sidra.ibge.gov.br/pesquisa/pnad

29. Instituto Brasileiro de Geografia e Estatística (IBGE). Pesquisa Nacional por Amostra de Domicílios Contínua - PNAD Contínua [Internet]. [acessado 2019 jul 27]. Disponível em: https://www.ibge.gov.br/estatisticas-novoportal/sociais/trabalho/9171-pesquisa-nacional-por-amostra-de-domicilios-continua-mensal. $\mathrm{html} ?=\& \mathrm{t}=\mathrm{o}$-que-e

30. Ipeadata. Brasil - Produto interno bruto (PIB): conceito de paridade do poder de compra (PPC) per capita [Internet]. 2020 [acessado $2020 \mathrm{fev}$ 10]. Disponível em: http://www.ipeadata.gov.br/Default.aspx

31. Manzano S. Quem é a classe trabalhadora brasileira? Blog da Boitempo [Internet]. 2019 [acessado 2020 fev 11]. Disponível em: https://blogdaboitempo.com. br/2019/12/03/quem-e-a-classe-trabalhadora-brasileira/ 
32. Rockett IR, Hobbs G, De Leo D, Stack S, Frost JL, Ducatman AM, Kapusta ND, Walker RL. Suicide and unintentional poisoning mortality trends in the United States, 1987-2006: Two unrelated phenomena? BMC Public Health 2010; 10.

33. Santos SA, Legay LF, Aguiar FP, Lovisi GM, Abelha L, Oliveira SP. Suicide and suicide attempts by exogenous poisoning in Rio de Janeiro, Brazil: Information analysis through probabilistic linkage | Tentativas e suicídios por intoxicação exógena no Rio de Janeiro, Brasil: Análise das informações através do linkage probab. Cad Saude Publica 2014; 30(5):1057-1066.

34. Classificação Brasileira de Ocupações (CBO). Portaria $n^{\circ}$ 397, de 09 de outubro de 2002 - 5.1.0. Aprova a Classificação Brasileira de Ocupações - CBO/2002, para uso em todo território nacional e autoriza a sua publicação. [Internet]. [acessado 2020 jan 20]. Disponível em: http://www.mtecbo.gov.br/cbosite/pages/ legislacao.jsf

35. Brasil. Ministério da Saúde (MS). Manual de Instruções para o preenchimento da Declaração de Óbito. Brasília: MS; 2011 [Internet]. [acessado 2020 jan 20]. Disponível em: http://svs.aids.gov.br/download/manuais/Manual_Instr_Preench_DO_2011_jan.pdf

36. Lima CRA, Schramm JMA, Coeli CM, Silva MEM. Review of data quality dimensions and applied methods in the evaluation of health information systems | Revisão das dimensões de qualidade dos dados e métodos aplicados na avaliação dos sistemas de informação em saúde. Cad Saude Publica 2009;25(10):2095-2109.

37. Cordeiro R, Peñaloza ER, Cardoso CF, Cortez DB, Kakinami E, Souza JJ, Souza MT, Fernandes RA, Guercia RF, Adoni T. Validity of information on occupation and principal cause on death certificates in Botucatu, São Paulo. Cad Saude Publica 1999; 15(4):719-728.

38. Rachiotis G, Stuckler D, McKee M, Hadjichristodoulou C. What has happened to suicides during the Greek economic crisis? Findings from an ecological study of suicides and their determinants (2003-2012). BMJ Open 5(3):e007295.

39. Branas CC, Kastanaki AE, Michalodimitrakis M, Tzougas J, Kranioti EF, Theodorakis PN, Carr BG, Wiebe DJ. The impact of economic austerity and prosperity events on suicide in Greece: A 30-year interrupted time-series analysis. BMJ Open 2015;5(1).

40. Kontaxakis V, Papaslanis T, Havaki-Kontaxaki B, Tsouvelas G, Giotakos O, Papadimitriou GN. Suicide in Greece: 2001-2011. Psychiatrike 2013; 24(3):170174.

41. Bando DH, Lester D. An ecological study on suicide and homicide in Brazil | Estudo ecológico sobre suicídio e homicídio no Brasil. Cien Saude Colet 2014; 19(4):1179-1189.

42. Jaen-Varas D, Mari JJ, Asevedo E, Borschmann R, Diniz E, Ziebold C, Gadelha A. The association between adolescent suicide rates and socioeconomic indicators in brazil: A 10-year retrospective ecological study. Brazilian J Psychiatry 2019;41(5):389-395.

43. Cuadrado C, Zitko P, Covarrubias T, Hernandez D, Sade C, Klein C, Gomez A. Association between adolescent suicide and sociodemographic factors in Chile: Cross-sectional ecological study. Crisis 2015; 36(4):281-290.
44. Gould MS, Greenberg T, Velting DM, Shaffer D. Youth suicide risk and preventive interventions: A review of the past 10 years. J Am Acad Child Adolesc Psychiatry 2003; 42(4):386-405.

45. Qin P, Agerbo E, Mortensen PB. Suicide risk in relation to socioeconomic, demographic, psychiatric, and familial factors: A national register-based study of all suicides in Denmark, 1981-1997. Am J Psychiatry 2003; 160(4):765-772.

46. Machado DB, dos Santos DN. Suicide in brazil, from 2000 to 2012 | Suicídio no Brasil, de 2000 a 2012. J Bras Psiquiatr 2015; 64(1):45-54

47. Macente LB, Zandonade E. Estudo da série histórica de mortalidade por suicídio no Espírito Santo (de 1980 a 2006). J Bras Psiquiatr 2011; 60(3):151-157.

48. Asevedo E, Ziebold C, Diniz E, Gadelha A, Mari J. Ten-year evolution of suicide rates and economic indicators in large Brazilian urban centers. Curr Opin Psychiatry 2018; 31(3):265-271.

49. Machado DB, Rasella D, Santos DN. Impact of income inequality and other social determinants on suicide rate in Brazil. PLoS One 2015; 10(4):e0124934.

50. Chang S-S, Stuckler D, Yip P, Gunnell D. Impact of 2008 global economic crisis on suicide: Time trend study in 54 countries. BMJ 2013; 347(7925).

51. Modrek S, Stuckler D, McKee M, Cullen MR, Basu S. A Review of Health Consequences of Recessions Internationally and a Synthesis of the US Response during the Great Recession. Public Health Reviews 2013(10). DOI: https://doi.org/10.1007/BF03391695

52. Antunes R, Praun L. A sociedade dos adoecimentos no trabalho. Serviço Soc Soc FapUNIFESP 2015; (123):407-427.

53. Alicandro G, Malvezzi M, Gallus S, La Vecchia C, Negri E, Bertuccio P. Worldwide trends in suicide mortality from 1990 to 2015 with a focus on the global recession time frame. Int J Public Health 2019; 64(5):785-795.

54. Chan CH, Caine ED, You S, Fu KW, Chang SS, Yip PSF. Suicide rates among working-age adults in South Korea before and after the 2008 economic crisis. J Epidemiol Community Health 2014; 68(3):246-252.

55. Corcoran P, Griffin E, Arensman E, Fitzgerald AP, Perry IJ. Impact of the economic recession and subsequent austerity on suicide and self-harm in Ireland: An interrupted time series analysis. Int J Epidemiol 2015; 44(3):969-977.

56. Madianos MG, Alexiou T, Patelakis A, Economou M. Suicide, unemployment and other socioeconomic factors: Evidence from the economic crisis in Greece. Eur J Psychiatry 2014; 28(1):39-49.

57. Tapia Granados JA, Rodriguez JM. Health, economic crisis, and austerity: A comparison of Greece, Finland and Iceland. Health Policy 2015;119(7):941-953.

58. Freire C, Koifman S. Pesticides, depression and suicide: A systematic review of the epidemiological evidence. Int J Hyg Environ Health 2013; 216(4):445-460.

59. Pires DX, Caldas ED, Recena MC. Intoxicações provocadas por agrotóxicos de uso agrícola na microrregião de Dourados, Mato Grosso do Sul, Brasil, no período de 1992 a 2002. Cad Saude Publica 2005; 21(3):804814. 
60. Meyer A, Koifman S, Koifman RJ, Moreira JC, Rezende Chrisman J, Abreu-Villaça Y. Mood disorders hospitalizations, suicide attempts, and suicide mortality among agricultural workers and residents in an area with intensive use of pesticides in Brazil. J Toxicol Environ Health 2010;73(13-14):866-877.

61. Mattei G, Ferrari S, Pingani L, Rigatelli M. Short-term effects of the 2008 Great Recession on the health of the Italian population: An ecological study. Soc Psychiatry Psychiatr Epidemiol 2014; 49(6):851-858.

Artigo apresentado em 20/04/2020

Aprovado em 21/07/2021

Versão final apresentada em 23/07/2021

Editores-chefes: Romeu Gomes, Antônio Augusto Moura da Silva 